International Journal of Biomedicine I June 2019 - Volume 9, Issue Suppl_1: Abstracts From the Second Russian International Conference "Cryo-electron microscopy 2019: achievements and prospects"

POSTER ABSTRACT PRESENTATIONS

SESSION TITLE: STRUCTURE OF MEMBRANE PROTEINS

DOI: 10.21103/IJBM.9.Suppl_1.P1

\title{
Abstract P-1: Analysis of the Structure of Serotonin 5-HT3 Receptor by X-Ray, CryoEM and MD Methods: Identification of Conformational State
}

\author{
Anna V. Popinako \\ A.N. Bach Institute of Biochemistry, Federal Research Center of Biotechnology of the Russian Academy \\ of Sciences, Moscow, Russia
}

Background: Ion channels form a varied class of integral membrane proteins involved in the regulation of fundamental cellular processes. The structural and functional diversity of ion channels, as well as their participation in the vital systems of the body causes an increased interest in their study. The importance of research of channels' structures is underscored by the identification of numerous "channelopathies", caused by ion channel mutations. However, the complex molecular architecture of eukaryotic ion channels, which include large non-membrane domains, is often an obstacle to structural studies by experimental methods. Thus, only epy atomic X-ray structure of the pentameric liganddependent mammalian channel of the serotonin 5-HT3 receptor (pdb id 4PIR) and the Cryo-EM structure (pdb id 6BE1) are known at the present. Based on the available experimental data on this channel, the conformational state of this channel cannot be determined. In this regard, the aim of this study was to compare and analyze the structure of serotonin 5-HT3 receptor and its models, built by modeling homology.

Methods: Structures with pdb id 2BG9, 4AQ9 for modeling 5-HT3 receptor closed and open conformations respectively were used as templates for modeling. We used the model of water TIP4P/2005 which suffciently well describes the liquid and crystal states. The cutoff radius for nonvalent interaction was $1.8 \mathrm{~nm}$. The calculations ran as an NPT ensemble. To maintain constant temperature and pressure, we used the stochastic dynamics thermostat and Berendsen barostat. We placed the ion channels molecules at the nodes of a hexagonal lattice and rotated them about their axes through random angles. The simulations ran in the software package Gromacs 4.6.7. We specified the initial velocities of atoms using a random number generator with the Maxwell distribution.

Results: The obtained models of open and closed channels of 5-HT3 receptor differ in the area of the internal threshold: the pore radius in this area is greater in open conformation models, compared with the closed conformation model and the structure of 5-HT3 receptor (4PIR). In the membrane part of the 5- 
HT3 receptor and the model of the closed conformation, the oxygen of the hydroxyl groups of threonine in the M2 helices form the area of the minimum radius of the pores.

Conclusion: According to the molecular dynamics data obtained by us, hydrated sodium ions are unable to pass through this section of the channel 5-HT3 of the receptor. Thus, the data obtained suggest that the structure of the 5-HT3 receptor ( $\mathrm{pdb}$ id 4PIR) is more consistent with the closed conformation. The work was carried out with the financial support of the Russian Foundation for basic research, agreement № 16-34-60252. The MD research has been carried out using the equipment of the shared research facilities of HPC computing resources at Lomonosov Moscow State University. This work structural analysis has been carried out using computing resources of the federal collective usage center Complex for Simulation and Data Processing for Mega-science Facilities at NRC "Kurchatov Institute"(ministry subvention under agreement RFMEFI62117X0016), http://ckp.nrcki.ru/.

Key Words: Ion channels $\bullet$ conformational state $\bullet$ modeling

International Journal of Biomedicine. 2019;9 Suppl 1: S16-17. doi: 10.21103/IJBM.9.Suppl_1.P1

C2019 International Medical Research and Development Corporation 